\title{
Consumo de tabaco entre pacientes con estomatitis aftosa recurrente
}

\section{Smoking habit in patients with recurrent aphthous stomatitis}

\author{
Eguia del Valle A*, Martínez-Conde Llamosas R*, López Vicente J*, \\ Uribarri Etxebarria A*, Aguirre Urizar JM*
}

\section{RESUMEN}

La Estomatitis Aftosa Recurrente (EAR) es una patología con una elevada prevalencia y una compleja etiopatogenia que aún no ha sido completamente esclarecida. El consumo de tabaco guarda una curiosa relación con esta patología ejerciendo un efecto protector cuyo origen no es bien comprendido y que ha sido objeto de controversia. En este trabajo se recogen los datos sobre consumo de tabaco en 171 pacientes con diagnóstico de EAR y se analiza su relación con los datos clínicos de dichos pacientes, atendiendo especialmente a la frecuencia, periodicidad, localización, tamaño y número de lesiones. Asimismo, en este trabajo se realiza una revisión de los aspectos más controvertidos de la relación entre el consumo de tabaco y la clínica de la EAR..

Palabras clave: Tabaco, estomatitis aftosa recurrente, aftosis oral recidivante, úlceras orales, consumo de tabaco.

\section{SUMMARY}

Recurrent aphtohus stomatitis (RAS) is a common type of ulcerative disease of the oral mucosa with a complex etiology that remains unclear. Smoking habit has a surprising, controversial and not well understood relationship with this disease, preventing the development of new lesions. In this work we present the results on tobacco consumption among 171 RAS patients. We also analyze the clinical aspects of these patients, especially those related to the frequency, location, number and size of the lesions. In this paper we also review the most important controversies on the relationship between the smoking habit and the clinical findings in RAU patients.

Key words: Tobacco, recurrent aphthous stomatitis, oral ulcers, smoking habit.

Fecha de recepción: 3 de diciembre de 2009.

Aceptado para publicación: 15 de diciembre de 2009.

* Medicina Bucal. Facultad de Medicina y Odontología. Universidad del País Vasco / EHU.

Eguia del Valle A, Martínez-Conde Llamosas R, López Vicente J, Uribarri Etxebarria A, Aguirre Urizar JM. Consumo de tabaco entre pacientes con estomatitis aftosa recurrentes. Av. Odontoestomatol 2011; 27 (3): 161-165.

\section{INTRODUCCIÓN}

El tabaco parece ejercer un paradójico efecto protector en los pacientes con Estomatitis Aftosa Recurrente (EAR) (1-6) Se ha constatado que la mayor parte de los pacientes con EAR son no fumadores $y$ que existe una menor prevalencia de EAR entre los pacientes fumadores que entre los no fumadores o fumadores moderados ( $<5$ cigarrillos/día) (1-4). Además, en numerosos pacientes se relaciona claramente el cese de este hábito con el desencadenamiento de brotes de lesiones, un empeoramiento de 
la evolución de la enfermedad o incluso el debut de la misma (1-3).

Diferentes hipótesis han sido postuladas para tratar de explicar este efecto. Se ha tratado de identificar, sin éxito por el momento, los componentes del tabaco que estarían implicados en el mismo, pero los resultados no han permitido identificarlos claramente (4). Algunos autores (2-4), consideran que el efecto protector que el tabaco parece ejercer en los pacientes con EAR, pudiera ser debido a que la hiperqueratinización que provoca en la mucosa oral, la protegería frente a pequeños traumatismos. No obstante, el tabaco no fumado y las tabletas con contenido en nicotina utilizadas en la terapia de deshabitualización del tabaquismo, parece asimismo ejercer el mismo efecto, aunque no esta claro aún a través de que mecanismo (2-6).

\section{MATERIAL Y MÉTODOS}

En el presente trabajo se recogieron los datos sobre consumo de tabaco en 171 pacientes con diagnóstico de Estomatitis Aftosa Recurrente procedentes de la Clínica de Medicina Bucal del Servicio Clínica Odontológica de la Universidad del País Vasco / EHU. La muestra de estudio estaba compuesta por 94 mujeres (55\%) y 77 hombres (45\%) con una edad media de 43,6 años ( \pm 12 años), comprendidos en un rango de edad entre 17 y 85 años. Todos ellos presentaban historia clínica compatible con un cuadro de EAR menor, mayor o herpetiforme, cumpliendo los criterios clínicos clásicos de la EAR (7-9), presentaban de un mínimo de 10 lesiones durante el periodo de 12 meses anterior a la realización del protocolo clínico y la exploración y en todos los casos se realizó un seguimiento clínico del paciente durante un mínimo de 12 meses. Tras el consentimiento informado, se realizó en todos los casos una encuesta sobre hábitos de consumo de tabaco y se recogieron los datos clínicos sobre las lesiones de todos los pacientes. Los pacientes fueron previamente diagnosticados de EAR menor, mayor o herpetiforme, atendiendo a la clasificación clínica clásica (1-10) y de EAR tipo I (periodos de tres meses o más sin lesiones), tipo II (periodos de 1 a 3 meses sin lesiones) o tipo III (lesiones continuadas) atendiendo a la clasificación de Bagán y cols. (9).

\section{RESULTADOS}

Un total de 32 pacientes (18,7\%) eran exfumadores y referían haber sufrido un aumento significativo en el número de lesiones tras cesar el consumo de tabaco. Además, en cinco de ellos (2,9\%) el debut de la enfermedad coincidió con el cese del consumo. Entre los pacientes exfumadores, 2 (1,2\%) habían sido fumadores ocasionales ( $<5$ cigarrillos diarios), $2(1,2 \%)$ de entre 5 y 10 cigarrillos diarios, $17(9,9 \%)$ habían fumado de 11 a 20 cigarrillos al día y otros $11(6,4 \%)$ habían sido fumadores de más de 20 cigarrillos diarios.

Por otra parte, 16 pacientes $(9,4 \%)$ eran fumadores en el momento actual. De ellos, 10 (5,8\%) eran fumadores ocasionales ( $<5$ cigarros diarios), $3(1,8 \%)$ fumaban entre 5 y 10 cigarrillos diarios, $2(1,2 \%)$ de 11 a 20 cigarrillos diarios, y tan solo $1(0,6 \%)$ era fumador de más de 20 cigarrillos diarios (Tabla 1) (Gráfico 1).

Estadísticamente, se observó una relación significativa entre el cese del consumo de tabaco y el tipo de lesiones que se producían. En la mayoría de casos eran lesiones de tipo menor $(p=0,01)$, continuas o de tipo $3(p=0,05)$, en numero igual o mayor de tres simultáneamente $(p=0,01)$ y en dos o más localizaciones simultáneas $(p=0,01)$

\section{DISCUSIÓN}

Numerosos estudios (1-6) han constatado el paradójico efecto "protector" del tabaco sobre la EAR La

\section{TABLA 1.- CONSUIMO DE TABACO ENTRE PACIENTES CON EAR}

\begin{tabular}{|l|r|c|r|c|}
\hline $\begin{array}{l}\text { Consumo de tabaco } \\
\text { (cigarrillos/día) }\end{array}$ & \multicolumn{2}{|c|}{$\begin{array}{c}\text { Fumador } \\
(\mathbf{n}=\mathbf{1 6})\end{array}$} & \multicolumn{2}{c|}{$\begin{array}{c}\text { Exfumador } \\
(\mathbf{n}=\mathbf{3 2})\end{array}$} \\
\hline & $\mathbf{n}$ & $\%$ & $\mathbf{n}$ & $\%$ \\
\hline$<5$ & 10 & 5,8 & 2 & 1,2 \\
$5-10$ & 3 & 1,8 & 2 & 1,2 \\
$11-20$ & 2 & 1,2 & 17 & 9,9 \\
$>20$ & 1 & 0,6 & 11 & 6,4 \\
\hline
\end{tabular}




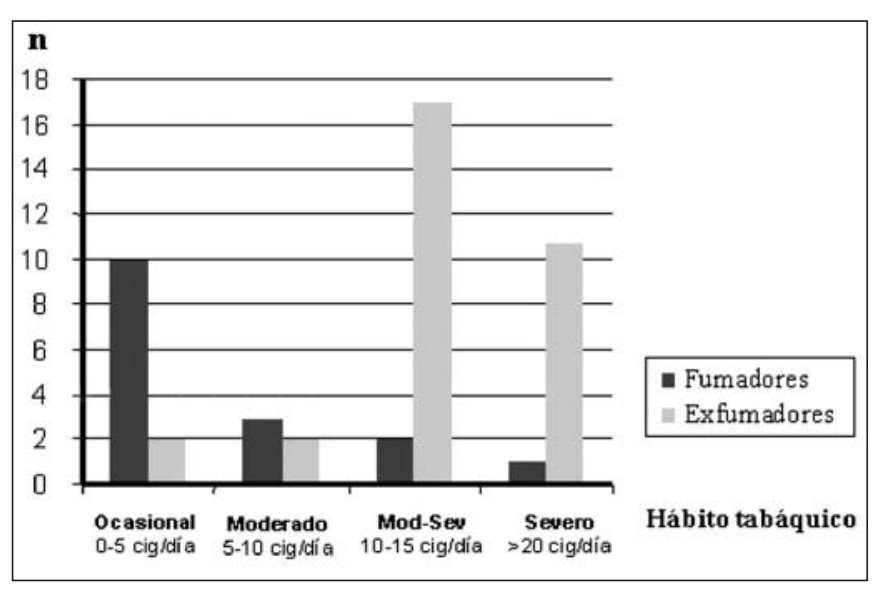

Gráf. 1. Relación con el tabaco.

prevalencia de fumadores entre los pacientes con EAR es mucho menor que si se compara con la prevalencia de fumadores entre muestras control de pacientes sanos o con los datos epidemiológicos sobre tabaquismo de la población que proceden.

En nuestro caso, sólo un 9,4\% de los pacientes con EAR eran fumadores, mientras que la prevalencia del tabaquismo en nuestra región se estima según diferentes estudios epidemiológicos, entre el 26 y el $34 \%$ para individuos mayores de 16 años de edad (10-13). Además, tras analizar en detalle los datos sobre nuestros pacientes fumadores con EAR, se observó que la mayoría correspondían a fumadores ocasionales o moderados ( $<5$ cig./día), que presentaban lesiones leves y ocasionales, del tipo 1 o 2 atendiendo a su frecuencia y en muy pocos casos del tipo 3 o en número igual o superior a tres lesiones simultáneas.

El mecanismo a través del cual el tabaco actúa como protector frente a la aparición de lesiones no se ha esclarecido por completo (2-5). De todas las hipótesis planteadas, la que goza de mayor consideración entre la comunidad científica, es la que sugiere que la hiperqueratinización provocada en la mucosa oral por el consumo de tabaco, la protegería de la acción de diferentes factores desencadenantes, especialmente de los factores traumáticos (3-6).

Sin embargo para algunos autores como Baron et al. (14) el efecto "protector" del tabaco frente a la aparición de lesiones en la EAR guardaría relación con otros de sus efectos, como es el efecto antiestrogénico, o las alteraciones en la producción de prostaglandinas o la estimulación de receptores colinérgicos y nicotínicos.

Además, autores como Grady et al. (15) o Bittoun (16), han observado que este efecto se produce también utilizando tabaco no fumado; tanto con el tabaco mascado como con los productos sustitutivos que se emplean para combatir la adicción al tabaco. Este hecho, arroja más dudas aún sobre el verdadero mecanismo a través del cual el tabaco actuaría como agente "protector" en la EAR.

Algunos trabajos como el de Cherubini et al. (17) han tratado de identificar sin éxito un componente concreto de entre los numerosos que contiene el tabaco al que hacer responsable en última instancia del efecto protector del tabaco en la EAR. Entre las sustancias más estudiadas, se halla el tiocianato, un ión liberado en la combustión del tabaco, que posee efectos perjudiciales propios, como la alteración en el metabolismo del yodo a nivel de la glándula tiroides $(18,19)$.

El tiocianato se puede utilizar como marcador biológico para medir el grado de exposición real al humo del tabaco, debido a su baja eliminación de los fluidos corporales y a su larga vida media de hasta 15 días, lo que hacen de él un marcador muy útil para estudiar exposiciones crónicas al humo del tabaco (17-19). No obstante, al comparar su concentración salivar entre pacientes con EAR en fase activa y en remisión, fumadores y no fumadores y pacientes sanos control no se ha podido establecer una relación clara, entre sus niveles salivales y la EAR (17).

En un interesante trabajo, Atkin et al. (20) comparan los niveles plasmáticos de cotinina en pacientes con EAR y población general. La cotinina es un componente del tabaco con una vida media larga en plasma y cuya determinación sirve para estimar objetivamente la cantidad de tabaco que consume una persona $(21,22)$. En este trabajo (20), se comprueba que el numero de fumadores entre los pacientes con EAR es sensiblemente menor que en la población general y que los niveles medios de cotinina de los pacientes con EAR que eran fumadores eran muy inferiores a la de los sujetos fumadores del grupo 
control. Todo esto pone de manifiesto que aún siendo fumadores, bien su hábito o bien su exposición real al tabaco era menor que la de los pacientes fumadores sin EAR.

En nuestro trabajo, entre los factores desencadenantes más frecuentes de la EAR, se encuentra el cese del consumo de tabaco en fumadores habituales. Casi una cuarta parte de nuestros pacientes refería que tras cesar el consumo de tabaco o durante los periodos en los que había cesado su consumo, habían sufrido un aumento llamativo en el número y frecuencia de lesiones aftosas o incluso que el comienzo de este proceso coincidía con este momento. Marakoglu et al. (23) en un reciente estudio han observado también este fenómeno. En su estudio comprobaron que el aumento en el número y frecuencia de las lesiones se producía especialmente a partir de la tercera semana del cese del consumo de tabaco y que este era significativamente más frecuente en los pacientes que no emplearon ningún tipo de medicación para cesar el consumo, que en aquellos que recurrieron a una terapia de reemplazo de nicotina.

El análisis de estos resultados nos ha permitido comprobar la existencia de una relación significativa entre el cese de consumo de tabaco y el tipo de lesiones que se producían. En estos casos, las lesiones eran de tipo menor, continuas, en numero igual o mayor de tres simultáneamente y en dos o más localizaciones simultáneas.

Podemos señalar a la vista de estos resultados sobre la relación entre el consumo de tabaco y la tipología de las lesiones en los pacientes con EAR y los datos aportados por los trabajos de otros autores (17-23) que el efecto del tabaco sobre la EAR podría guardar una relación dosis-dependiente.

\section{BIBLIOGRAFÍA}

1. Axell T, Henricsson V. Association between recurrent aphtous ulcers and tobacco habits. Scand J Dent Res 1985;93:239-42.

2. Baron JA. Beneficial effects of nicotine and cigarrette smoking: the real, the possible and the spurious. Br Med Bull 1996;52:58-73.
3. Grady D, Ernster VL, Stillman L, Greenspan J. Smokeless tobaco use prevents aphtous stomatitis. Oral Surg Oral Med Oral Pathol Oral Radiol Endod 1992;74:463-5.

4. Cherubini K, Lorandi CS, Krapf SM, Sousa FR, Yurgel LS, Figueiredo MA, Salud FG. Association between recurrent aphthous stomatitis and salivary thiocyanate levels. J Oral Sci 2006;48: 153-6.

5. Tuzun B, Wolf R, Tuzun Y, Serdaroglu S. Recurrent aphthous stomatitis and smoking. Int J Dermatol 2000;39:358-60.

6. Shapiro S, Olson DL, Chelimi SJ. The association between smoking and aphthous ulcers. Oral Surg Oral Med Oral Pathol 1970;30:624-30.

7. Ship JA. Recurrent aphtous stomatitis. An update. Oral Surg Oral Med Oral Pathol Oral Radiol Endod 1996;81:141-7.

8. Esparza G, Cerero R, Moreno LA. Estomatitis aftosa recurrente. Gac Dent 2000;104:44-52.

9. Bagán JV, Sanchis JM, Millán MA, Peñarrocha M, Silvestre FJ. Recurrent aphtous stomatitis. A study of the clinical characteristics of lesions in 93 cases. J Oral Pathol Med 1991;20:395-7.

10. Saiz I, Rubio J, Espiga I, Alonso de la Iglesia B, Blanco J, Cortés M, Cabrera J. Plan nacional de prevención y control del tabaquismo. Rev Esp Salud Pública 2003;77:441-73.

11. Banegas JR, Díaz L, Rodriguez-Artalejo F, González J, Pérez G, Regadera A, Villar Alvarez F. Mortalidad atribuible al tabaquismo en España en 1998. Med Clin (Barc) 2001;117:692-4.

12. Comité Nacional para la Prevención del Tabaquismo. Libro Blanco sobre el Tabaquismo en España. Barcelona: Glosa; 1998.

13. Gil E, Robledo T, Rubio JM, Bris MR, Espiga I, Saiz I. Prevalencia del consumo de tabaco en los profesionales sanitarios del Insalud 1998, España. Prev Tab 2000;2:22-31. 
14. Baron JA. Beneficial effects of nicotine and cigarrette smoking: the real, the possible and the spurious. Br Med Bull 1996;52:58-73.

15. Grady D, Ernster VL, Stillman L, Greenspan J. Smokeless tobaco use prevents aphtous stomatitis. Oral Surg Oral Med Oral Pathol Oral Radiol Endod 1992;74:463-5.

16. Bittoun R. Recurrent aphthous ulcers and nicotine. Med J Aust 1991;154:471-2.

17. Cherubini K, Lorandi CS, Krapf SM, Sousa FR, Yurgel LS, Figueiredo MA, Salud FG. Association between recurrent aphthous stomatitis and salivary thiocyanate levels. J Oral Sci 2006;48:153-6.

18. Yang P, Wei W, Tao C. Determination of trace thiocyanate with nano-silver coated multi-walled carbon nanotubes modified glassy carbon electrode. Anal Chim Acta 2007;585:331-6.

19. Scherer G. Carboxyhemoglobin and thiocyanate as biomarkers of exposure to carbon monoxide and hydrogen cyanide in tobacco smoke. Exp Toxicol Pathol 2006;58:101-24.

20. Atkin PA, Xu X, Thornhill MH. Minor recurrent aphthous stomatitis and smoking: an epide- miological study measuring plasma cotinine. Oral Dis 2002;8:173-6.

21. Benowitz NL, Jacob P 3rd, Herrera B.Nicotine intake and dose response when smoking reduced-nicotine content cigarettes. Clin Pharmacol Ther 2006;80:703-14.

22. Blackford AL, Yang G, Hernandez-Avila M, Przewozniak K, Zatonski W, Figueiredo V, AvilaTang E, Ma J, Benowitz NL, Samet JM. Cotinine concentration in smokers from different countries: relationship with amount smoked and cigarette type. Cancer Epidemiol Biomarkers Prev 2006;15:1799-804.

23. Marakoglu K, Sezer RE, Toker HC, Marakoglu I. The recurrent aphthous stomatitis frequency in the smoking cessation people. Clin Oral Investig 2007;11:149-53.

\section{CORRESPONDENCIA}
A. Eguia del Valle
Barrio Sarriena, s/n
48940 Leioa (Vizcaya)

E-mail: aeguiadelvalle@yahoo.es 